\title{
A Scalable Correlator Architecture Based on Modular FPGA Hardware, Reuseable Gateware, and Data Packetization
}

\author{
Aaron Parsons, Donald Backer, and Andrew Siemion \\ Astronomy Department, University of California, Berkeley, CA \\ aparsons@astron. berkeley.edu \\ Henry Chen and Dan Werthimer \\ Space Science Laboratory, University of California, Berkeley, CA \\ Pierre Droz, Terry Filiba, Jason Manley ${ }^{1}$, Peter McMahon ${ }^{1}$, and Arash Parsa \\ Berkeley Wireless Research Center, University of California, Berkeley, CA \\ David MacMahon, Melvyn Wright \\ Radio Astronomy Laboratory, University of California, Berkeley, CA
}

\begin{abstract}
A new generation of radio telescopes is achieving unprecedented levels of sensitivity and resolution, as well as increased agility and field-of-view, by employing highperformance digital signal processing hardware to phase and correlate large numbers of antennas. The computational demands of these imaging systems scale in proportion to $B M N^{2}$, where $B$ is the signal bandwidth, $M$ is the number of independent beams, and $N$ is the number of antennas. The specifications of many new arrays lead to demands in excess of tens of PetaOps per second.

To meet this challenge, we have developed a general purpose correlator architecture using standard 10-Gbit Ethernet switches to pass data between flexible hardware modules containing Field Programmable Gate Array (FPGA) chips. These chips are programmed using open-source signal processing libraries we have developed to be flexible, scalable, and chip-independent. This work reduces the time and cost of implementing a wide range of signal processing systems, with correlators foremost among them, and facilitates upgrading to new generations of processing technology. We present several correlator deployments, including a 16-antenna, 200-MHz bandwidth, 4-bit, full Stokes parameter application deployed on the Precision Array for Probing the Epoch of Reionization.
\end{abstract}

Subject headings: Astronomical Instrumentation

\footnotetext{
${ }^{1}$ Affiliated with Karoo Array Telescope, Cape Town, South Africa
} 


\section{Introduction}

Radio interferometers, which operate by correlating the signals from two or more antennas, have many advantages over traditional single-dish telescopes, including greater scalability, independent control of aperture size and collecting area, and self-calibration. Since the first digital correlator built by Weinreb (Weinreb 1961), the processing power of these systems has been tracking the Moore's Law growth of digital electronics. The decreasing cost per performance of these systems has influenced the design of many new radio antenna array telescopes. Some next-generation array telescopes at meter, centimeter and millimeter wavelengths are: the LOw Frequency ARray (LOFAR), the Precision Array for Probing the Epoch of Reionization (PAPER), the Murchison Widefield Array (MWA), the Long Wavelength Array (LWA), the Expanded Very Large Array (EVLA), the Allen Telescope Array (ATA), the Karoo Array Telescope (MeerKAT), the Australian Square Kilometer Array Demonstrator (ASKAP), the Atacama Large Millimeter Array (ALMA). and the Combined Array for Research Millimeter-wave Astronomy (CARMA). This paper presents

a novel approach to the intense digital signal processing requirements of these instruments that has many other applications to astronomy signal processing.

While each generation of electronics has brought new commodity data processing solutions, the need for high-bandwidth communication between processing nodes has historically lead to specialized system designs. This communication problem is particularly germane for correlators, where the number of connections between nodes scales with the square of the number of antennas. Solutions to date have typically consisted of specialized processing boards communicating over custom backplanes using non-standard protocols. However, such solutions have the disadvantage that each new generation of digital electronics requires expensive and time-consuming investments of engineering time to re-solve the same connectivity problem. Redesign is driven by the same Moore's Law that makes digital interferometry attractive, and is not unique to the interconnect problem; processors such as Application-Specific Integrated Circuits (ASICs) and Field Programmable Gate Arrays (FPGAs) also require redesign, as do the boards bearing them, and the signal processing algorithms targeting their architectures.

Our research is aimed at reducing the time and cost of correlator design and implementation. We do this, firstly, by developing a packetized communication architecture relying on industrystandard Ethernet switches and protocols to avoid redesigning backplanes, connectors, and communication protocols. Secondly, we develop flexible processing modules that allow identical boards to be used for a multitude of different processing tasks. These boards are applicable to general signal processing problems that go beyond correlators and even radio science to include, e.g., ASIC design and simulation, genomics, and research into parallel processor architectures. General purpose hardware reduces the number of boards that have to be redesigned and tested with each new generation of electronics. Thirdly, we create parametrized signal processing libraries that can easily be recompiled and scaled for each generation of processor. This allows signal processing systems to quickly take advantage of the capabilities of new hardware. Finally, we employ an extension of a Linux kernel to interface between CPUs and FPGAs for the purposes of testing and control, 
presenting a standard file interface for interacting with FPGA hardware.

This paper begins with a presentation of the new correlator design architecture in \$2 The hardware to implement this architecture follows in 93 , and the FPGA gateware used in the hardware is summarized in $\$ 4$. Issues concerning system integration are given in $\$ 5$, and performance characterization of subsystems are given in $\$ 6$. Results from our first deployments of the packetized correlator are displayed in \$7. Our final section summarizes our progress and points to a number of directions we are pursuing for the next generation of scalable correlators based on modular hardware, reuseable gateware and data packetization. An appendix gives a glossary of technical acronyms since this paper makes heavy use of abbreviated terms.

\section{A Scalable, Asynchronous, Packetized FX Correlator Architecture}

Correlators integrate the pairwise correlation between complex voltage samples from polarization channels of array antenna receivers at a set of frequencies. Once instrumental effects have been calibrated and removed, the resultant correlations (called visibilities) represent the self-convolved electric field across an aperture sampled at locations corresponding to separations between antennas. These visibilities can be used to reconstruct an image of the sky by inverting the interferometric measurement equation:

$$
V_{\nu}(u, v)=\iint G_{i, \nu} G_{j, \nu}^{*} I_{\nu}(\ell, m) e^{-2 \pi i\left(u \ell+v m+w\left(\sqrt{1-\ell^{2}-m^{2}}-1\right)\right)} d \ell d m
$$

$I_{\nu}$ represents the sky brightness in angular coordinates $(\ell, m)$, and $(u, v, w)$ correspond to the separation in wavelengths of an antenna pair relative to a pointing direction. For antennas with separate polarization feeds, cross-correlation of polarizations yields components of the four Stokes parameters that characterize polarized radiation, here defined in terms of linear polarizations $(\|, \perp)$ for all pairs of antennas $A$ and $B$ (Rybicki \& Lightman 1979):

$$
\begin{array}{ll}
I=A_{\|} B_{\|}^{*}+A_{\perp} B_{\perp}^{*} & Q=A_{\|} B_{\|}^{*}-A_{\perp} B_{\perp}^{*} \\
U=A_{\|} B_{\perp}^{*}+A_{\perp} B_{\|}^{*} & V=A_{\|} B_{\perp}^{*}-A_{\perp} B_{\|}^{*}
\end{array}
$$

I measures total intensity, $\mathrm{V}$ measures the degree of circular polarization, and $\mathrm{Q}$ and $\mathrm{U}$ measure the amplitude and orientation of linear polarization.

The problem of computing pairwise correlation as a function of frequency can be decomposed two mathematically equivalent but architecturally distinct ways. The first architecture is known as "XF" correlation because it first cross-correlates antennas (the "X" operation) using a time-domain "lag" convolution, and then computes the spectrum (the " $\mathrm{F}$ " operation) for each resulting baseline using a Discrete Fourier Transform (DFT). An alternate architecture takes advantage of the fact that convolution is equivalent to multiplication in Fourier domain. This second architecture, called "FX" correlation, first computes the spectrum for each individual antenna (the F operation), and then multiplies pairwise all antennas for each spectral channel (the X operation). An FX correlator 
has an advantage over XF correlators in that the operation that scales as $O\left(N^{2}\right)$ with the number of antennas, $\mathrm{N}$, is a complex multiplication as opposed to a full convolution in an XF correlator (D'Addario 2001; Yen 1974).

Though there are mitigating factors (such as bit-growth for representing the higher dynamic range of frequency-domain data) that favor XF correlators for small numbers of antennas (Thompson et al. 2001), FX correlators are more efficient for larger arrays. Since scalability to large numbers of antennas is one of the primary motivations of our correlator architecture, we have chosen to develop FX architectures exclusively.

\subsection{Scalability With Number of Antennas and Bandwidth}

The challenge of creating a scalable FX correlator is in designing a scalable architecture for factoring the total computation into manageable pieces and efficiently bringing together data in each piece for computation. Traditionally, the spectral decomposition (in F engines) has been scaled to arbitrary bandwidths by using analog mixers and filters to divide the operating band of each antenna into the widest subbands that can be processed digitally using existing technology. Within correlation of a given subband, the complexities of computation and of data distribution both scale linearly with bandwidth and quadratically with the number of antennas. It is imperative that the arrangement of cross-multiplication engines (hereafter referred to as $\mathrm{X}$ engines) minimize data replication/retransmission, even as X engines expand to encompass many boards. Fortunately, each frequency channel of an FX correlator is computationally independent, providing a natural boundary for dividing computation among processing nodes.

Figure 1 illustrates a simplistic architecture for an FX correlator that takes advantage of the computational independence of channels to avoid unnecessary data transmission; the total X computation has been factored into $\mathrm{X}$ engines that cross-multiply all antenna pairs for a single frequency channel. This architecture is overly simplistic, since an X engine's performance can be equated to an aggregate input bandwidth that it can handle. For the sake of efficiency, an X engine processor should receive as many channels as it has capacity to process. In this case, the number of $\mathrm{X}$ engines is given by:

$$
\# \mathrm{X} \text { Engines }=\frac{(\text { Antenna Bandwidth }) \times(\# \text { Antennas })}{\mathrm{X} \text { Engine Processing Bandwidth }}
$$

Multiplexing channels into $\mathrm{X}$ engines makes cross-multiplication complexity independent of the number of channels. There are three potential bottlenecks for scaling this architecture: the complexity of interconnecting $\mathrm{F}$ engines and $\mathrm{X}$ engines, the bandwidth into individual $\mathrm{X}$ engines, and the amount of computation in an $\mathrm{X}$ engine relative to the size of a processing chip/board/system. Each of these bottlenecks warrants further discussion.

The potential bottleneck of connecting $N$ antenna-based $\mathrm{F}$ engines to $M$ channel-based $\mathrm{X}$ engines is highlighted by the criss-crossed lines in Figure 1. Historically, this bottleneck has been 
addressed with custom backplanes and transmission protocols. However, our group has taken the novel approach of using high-performance, commercially available, 10-Gbit/s Ethernet (10GbE) switches to solve this problem. As will be discussed, these switches currently have the bandwidth and switching capacity to handle large correlators, and represent a negligible fraction of the total cost of correlator hardware. Furthermore, switching technology is driven by commercial applications and by Moore's Law, making it likely that future switches will continue increasing in number of ports and bandwidth per port.

A second potential bottleneck concerns how data rates and numbers of $\mathrm{X}$ engines scale with antenna bandwidth. It is important that we consider various bandwidth cases, owing to the variety of science applications driving large, next-generation systems. For example, correlators for large arrays of low-bandwidth antennas will need to multiplex data into higher bandwidth processors, while arrays with larger bandwidths will face the opposite problem. In our architecture, we make the reasonable assumption that the number of frequency channels always exceeds the number of antennas. This assumption ensures that the per-port bandwidth into an $\mathrm{X}$ engine never exceeds what is transmitted per antenna. Multiple channels may then be mapped into an X engine up to its computational capacity (allowing efficient resource utilization for low-bandwidth arrays), and additional X engines may be added for high-bandwidth applications. Antenna bandwidths requiring transmission above $10 \mathrm{Gbits} / \mathrm{s}$ can be accommodated by connecting F engines to multiple 10GbE ports. Frequency channels are then assigned to each port, which connect separate switches and sub-networks of X engines. In this way, bandwidths may be scaled up to the transmission capability of an F processor by increasing the number of subnets, and not switch complexity.

The third and final potential bottleneck concerns how the sizes of individual X engines scales with the number of antennas. Both large and small numbers of antennas pose scaling problems. The size of an $\mathrm{X}$ engine responsible for computing all baseline cross-multiples with a fixed input data rate scales as $O(N)$, while the number of $\mathrm{X}$ engines required to accommodate the expanding data bandwidth with increasing numbers of antennas also scales as $O(N)$, accounting for the $O\left(N^{2}\right)$ scaling of computing in a correlator. For sufficiently large $N$, the size of an $\mathrm{X}$ engine can exceed the size of any processing chip or board. Our solution has been to develop an $\mathrm{X}$ engine whose pipelined architecture allows it to be split across multiple processors with simple point-to-point connectivity. This allows many processors to be chained together from a switch port to meet the computational demands of an $\mathrm{X}$ engine. Scaling to small $N$ is equally challenging, because the aggregate correlator bandwidth decreases as $O(N)$, while computational complexity scales down as $O\left(N^{2}\right)$. As a result, we can find that the number of $\mathrm{X}$ engines that fit onto a chip/board exceeds the rate at which data can be received. The threshold where this problem is encountered can be changed by designing processors with greater connectivity, but once hardware is fixed, there is no other recourse but to accept a certain inefficiency for low numbers of antennas. While this is a fundamental limitation of our architecture, the cost of small correlators is typically dominated by development (not hardware), so a certain architectural inefficiency can be accommodated for the savings it affords in development time. 


\subsection{Globally Asynchronous Locally Synchronous Systems}

Packetized data transmission simplifies the cross-connect problem inherent to correlators, but this comes at the price of global synchronicity. Packetized communication is fundamentally asynchronous: data can arrive scrambled, delayed, or not at all. Locally-synchronous X engine processing must therefore transition from being timing-driven (with throughput tied to an FPGA clock, for example) to being asynchronously data-driven. Though data buffers and control signals complicate development, Globally Asynchronous Locally Synchronous (GALS) design facilitates system integration and leads to robust design (Chapiro 1984; Plana et al. 2007). Processors run at clock rates above the data rate, using local oscillators that can drift with temperature. By allowing for non-transmission of data, individual components can fail without causing global failure-an important feature for large systems where components may fail regularly during operation. GALS design also insulates processing architectures from decisions regarding sample rates and antenna bandwidths, allowing for greater operational flexibility. Finally, individual processing elements may be redesigned and upgraded in a GALS system without affecting the overall architecture, facilitating early adoption of new technology.

Data-driven processing on locally synchronous processors like FPGAs requires controlling propagation through the processing pipeline. However, routing control signals to every multiplier, accumulator, and logic element in a pipeline can lead to excessive routing and gating demands. To avoid this, we have implemented a window-based processing architecture for algorithms where the results derived from one set of data samples are computationally independent from the next. In this architecture, processing elements are allowed to run freely at their native rate without being enabled/disabled, but are only provided data when an entire window of data has been buffered. These windows of data are provided synchronously with the inherent window boundaries of the processing element, and an entire output window is flagged as valid. Internally, a processor processes both valid and invalid data-it is only the external buffering system that keeps track of data validity. This technique is applicable to many common operators such as cross-multipliers, DFTs, and accumulators. Finite Impulse Response (FIR) filtering is an operation notable for not being window-based.

\subsection{Example Applications}

Perhaps the best method for demonstrating the flexibility and scalability of our correlator architecture is through example applications. To illustrate techniques for using hardware and ports efficiently, we will map processing into fictitious hardware that corresponds roughly in capability

to the CASPER (Center for Astronomy Signal Processing and Engineering Research) 1 hardware discussed in Section 3 ,

\footnotetext{
${ }^{1}$ http://casper.berkeley.edu
} 
Our first example (Fig. 2) illustrates an antenna signal bandwidth sufficiently low so that data from 2 polarization channels of 2 antennas can be transmitted over one 10GbE connection. Assuming that the number of antennas evenly divides the number of frequency channels, and that the processing bandwidth of an X engine matches the data bandwidth of one antenna, there will be the same number of $X$ engines as $F$ engines, and each $X$ engine will receive $1 / N^{\text {th }}$ of the total bandwidth, where $\mathrm{N}$ is the number of antennas. $\mathrm{F}$ engine transmission and $\mathrm{X}$ engine reception are combined on a single port to make use of the bi-directionality of 10GbE. This optimization halves the size of the switch needed. Multiple X processors can be chained together from a single $10 \mathrm{GbE}$ port using point-to-point connections. For cases where the number of antennas does not evenly divide the number of frequency channels, one can adjust packet transmission to drop remainder channels so that the band may be equally divided among $\mathrm{X}$ engines.

A second example (Fig. 3) illustrates a case where the bandwidth from a single $\mathrm{F}$ engine exceeds the transmission capacity of a 10GbE link. Here, data can be split by frequency channel across two ports. Since different channels are never cross-multiplied, each of these links goes to a separate subnet of switched X engines. Thus, two smaller (and often less expensive per port) switches may be substituted for one large one. Each $\mathrm{X}$ engine still receives the same bandwidth as in the previous example, although this now represents a smaller fraction of the total bandwidth. Note that the same $\mathrm{X}$ processor used in the first example functions here without modification. Only the number of $\mathrm{X}$ engines and the transmission pattern has changed.

A final example (Fig. 4) explores the case where the capacity of an X processor and a $10 \mathrm{GbE}$ link both exceed the data bandwidth. In this case, multiple F engines can (but do not have to) be chained together to minimize the number of switched ports. As should be the case, only half as many $\mathrm{X}$ engines (as compared to Fig. 2) are necessary for a given number of antennas. X processors operate in the same configuration as before, oblivious to changes in $\mathrm{F}$ engines.

These examples highlight the flexibility of the hardware and gateware for targeting a number of applications. One shortcoming they also illustrate is how the cabling between components differs for different bandwidths. Therefore the different bandwidth operations are not as easily reconfigured as might be desired for varying science goals on a given telescope. Research is ongoing to improve the rapid reconfigurability that is an essential specification for the most general radio interferometer array applications.

\section{Modular, FPGA-based Processing Hardware}

A flexible and scalable correlator architecture is of limited use without equally dynamic processing hardware that can support a variety of configurations. FPGAs provide a unique combination of flexibility and performance that make them well-suited for moderate-scale signal processing applications such as correlators and spectrometers (Parsons et al. 2006). A primary goal of the CASPER group has been development of multipurpose processing modules that can be of general use to the 
astronomy signal processing community, and beyond. We seek to minimize the effort of redesigning and upgrading hardware by modularizing processing hardware, by minimizing the number of different modules in a system, and by employing industry-standard interconnection protocols.

Hardware modularity is the idea that boards should have consistent interfaces in order to be connectible with an arbitrary number of heterogeneous components to meet the computing needs of an application ("computing by the yard"), and that upgrading/revising a component does not change the way in which components are combined in the system. Minimization of hardware reproduction costs is often used to motivate the design of specialized hardware for large-scale correlators. However, the longer development times inherent to such solutions, and the necessity of targeting specific components from the outset, suggest that a modular solution, initiated nearer to the deployment date, will employ newer technology that costs less and uses less power per operation. The predicted economy of mass-producing specially-designed hardware must be tempered by its expected devaluation by Moore's Law over the course of correlator development. This devaluation makes the argument that hardware modularity can reduce the overall system cost, even for largescale systems, by reducing development time.

In current correlator systems, we rely on two CASPER FPGA-based processing boards; Internet Break-Out Boards (IBOBs) are generally used for implementing per-antenna $\mathrm{F}$ engine processing, and second-generation Berkeley Emulation Engines (BEE2s) implement X engine processing. Work is progressing on a new board, the Reconfigurable Open Architecture for Computing Hardware $(\mathrm{ROACH})$, that will provide a single-board solution to both $\mathrm{F}$ and $\mathrm{X}$ processing.

IBOBs (Fig. 5) can interface to two Analog-to-Digital Converter (ADC) boards, each capable of digitizing two streams at 1 Gsamples/sec or a single stream at 2 Gsamples/sec using an Atmel AT84AD001B dual 8-bit ADC chip. This data is processed by a Xilinx XC2VP50 FPGA containing $23218 \times 18$-bit multipliers, two PowerPC CPU cores, and over 53,000 logic cells. Two ZBT SRAM chips provide 36 Mbits of extra buffering, and two 10GbE-compatible CX4 connectors provide a standard interface for connecting to other boards, switches, and computers. A detailed discussion of ADC signal fidelity is presented in Section 6. We are developing a second ADC board that allows four signal sampling at 200 Msample/sec.

The BEE2 board Chang et al. (2005) (Fig. 5) was originally designed for high-end reconfigurable computing applications such as ASIC design, but has been conscripted for astronomy applications in a collaboration between the BWRC2 the UC Berkeley Radio Astronomy Laboratory, and the UC Berkeley SETI group. The $500 \mathrm{Gops} / \mathrm{sec}$ of computational power in the BEE2 is provided by 5 Xilinx XC2VP70 Virtex-II Pro FPGAs, each containing 328 multipliers, two PowerPC CPU cores capable of running Linux, and over 74,000 configurable logic cells. Each FPGA connects to $4 \mathrm{~GB}$ of DDR2-SDRAM, and four 10GbE-compatible CX4 connectors, and all FPGAs share a 100-Mbps Ethernet port. The size and connectivity of the BEE2 board make it suitable

\footnotetext{
${ }^{2}$ Berkeley Wireless Research Center http://bwrc.eecs.berkeley.edu
} 
for implementing $\mathrm{X}$ engine processing in our correlator architecture.

The ROACH board is being developed in collaboration with MeerKAT and NRAO 3 and is scheduled for release in the third quarter of 2008. It is intended as a replacement for both IBOB and BEE2 boards. A single Xilinx Virtex-5 XC5VSX95T FPGA containing 94,000 logic cells and 640 multiplier/accumulators provides $400 \mathrm{Gops} / \mathrm{sec}$ of processing power and is connected to a separate PowerPC 440EPx processor with a $1 \mathrm{GbE}$ network connection. The board contains 4 GB of DDR2 DRAM and two 36Mbit QDR SRAMs, four 10GbE-compatible CX4 connectors, and two interfaces that allow the use of the current ADC boards, or a new 3 Gsamples/sec (6 Gsamples/sec dual-board interleaved) ADC. The scale, economy, and peripheral interfaces of this board will make it appropriate for both $\mathrm{F}$ and $\mathrm{X}$ engine processing, and will enable a single-board correlator architecture.

\section{Gateware}

Efficient, customizable signal processing libraries are another important component of a flexible and scalable correlator architecture. Towards this goal, our group has designed a set of open-source libraries 4 for the Simulink/Xilinx System Generator FPGA programming language. These libraries abstract chip-specific components to provide high-level interfaces targeting a wide variety of devices. Signal processing blocks in these libraries are parametrized to scale up and down to arbitrary sizes, and to have selectable bit widths, latencies, and scaling. Though the design principles of parametrization and scalability have added complexity to the initial design of these libraries, it dramatically enhances their applicability and potential for longevity as hardware evolves. It also decreases testing time by allowing developers to debug scale models of systems that derive from the same parametrization code and are behaviorally similar to larger systems. In this section, we present several components of our libraries vital to the design of flexible correlators.

\subsection{A Digital Down-Converter}

The rising speed of ADCs has enabled digitization to occur increasingly early in the antenna receiver chain. We are thus replacing analog electronics commonly known as intermediate frequency processor (gain, band definition) and baseband mixer (conversion to zero frequency and filtering). There are numerous advantages to doing this. Digital mixing allows dynamically selecting an operating frequency within the digitized band while ensuring perfect sine-cosine phasing in the local oscillator (LO) mixing frequency. Digitizing a wider bandwidth than will be ultimately processed

\footnotetext{
${ }^{3}$ The National Radio Astronomy Observatory (NRAO) is owned and operated by Associated Universities, Inc. with funding from the National Science Foundation

${ }^{4}$ Available at http://casper.berkeley.edu
} 
makes analog filtering less critical; inexpensive filters with slow roll-offs can be used, and passband rippling can be corrected. Finally, digital filtering allows flexibility and control in selecting passband shapes and adjusting fine delays. One can even split out several bands from the same signal. The issue of quantization levels and other digital artifacts needs to be carefully addressed.

Our library provides a digital down-conversion core with a runtime-selectable mixing frequency. Using a discretely sampled sine wave in an addressable lookup table, we can approximate nearly any mixing frequency by rounding a wide accumulation register (incremented every clock) to the nearest address in the lookup table. Digital sine waves have an accuracy dictated by the number of bits used to represent a value; a lookup table need only have enough samples to achieve comparable accuracy. The fact that the derivative of $\sin (x)$ reaches a maximum magnitude of 1 allows the sampling interval of a sine wave to be simply equated to the accuracy of a coefficient over that time interval. As a result, a lookup table only need be addressed with the same bit-width as the sample width to implement an arbitrary mixing frequency.

Our library also contains a decimating FIR filter. Digital filters have advantages over analog filters by being reprogrammable and by providing exact, calculable passbands. This filter is often used for suppressing harmonics of the mixing frequency and for steepening the rolloff of cheaper analog filters, but it has also been relied upon for implementing IF sub-band selection digitally. In practice, one must weigh the need for performance and flexibility against the cost of FPGA resources compared to analog filters. As an example, the response of the FIR filter used in various correlator designs is shown in Figure 6. Since the exact shape of this filter can be calculated, it is possible to remove passband ripple post-channelization because of the large dynamic range available in output of our FFT core.

\subsection{A Polyphase Filter Bank Front-End}

The Polyphase Filter Bank (PFB) (Crochiere \& Rabiner 1983; Vaidyanathan 1990) is an efficient implementation of a bank of evenly spaced, decimating FIR filters. The PFB algorithm decomposes these filters into a single polyphase convolution followed by a DFT. Since DFTs have been highly optimized algorithmically, this results in an extremely efficient implementation. Equivalently, the PFB may be regarded as an improvement on the Fast Fourier Transform (FFT) that uses a front-end polyphase FIR filter to improve the frequency response of each spectral channel (Fig. 7). This improvement comes at the cost of buffering an additional window of samples and adding a complex cross-multiplication for each additional tap in the polyphase FIR. This PFB implementation has seen widespread use in the astronomy community in $21 \mathrm{~cm}$ hydrogen surveys (Heiles et al. 2004), pulsar surveys (Demorest et al. 2004), antenna arrays (Bradley et al. 2005), Very Long Baseline Interferometry, and other applications.

Our core is parametrized to use selectable windowing functions, allowing adjustment of the out-of-band rejection and passband ripple/rolloff. Blackman and Tukey (Blackman \& Tukey 1958) 
provides a summary of the characteristics and trade-offs of various windows. Each polyphase FIR tap, at the cost of increased buffering and additional multipliers, increases filter steepness by adding samples (in increments of the number of channels) to the time window used in the PFB. For fixedpoint implementations, a practical upper limit to the number of PFB taps is set by the number of bits used to represent filter coefficients; the sinc function's $1 / x$ tapering ceases to be representable when $\pi T>\pi+2^{B+1}$ where $T$ is the number of taps, and $B$ is the coefficient bit width. Finally, the width of a PFB channel is tunable by adjusting the period of the sinc function, forcing adjacent bandpass filters to overlap at a point other than the $-3 \mathrm{~dB}$ point. Note that this causes power to no longer be conserved in the Fourier transform operation.

\subsection{A Bandwidth-Agile Fast Fourier Transform}

The computational core of our FFT library is an implementation of a radix-2 biplex pipelined FFT (Rabiner \& Gold 1975) capable of analyzing two independent, complex data streams using a fraction of the FPGA resources of commercial designs (Dick 2000). This architecture takes advantage of the streaming nature of ADC samples by multiplexing the butterfly computations of each FFT stage into a single physical butterfly core. When used to analyze two independent streams, every butterfly in this biplex core outputs valid data every clock for $100 \%$ utilization efficiency.

The need to analyze bandwidths higher than the native clock rate of an FPGA led us to create a second core that combines multiple biplex cores with additional butterfly cores to create an FFT that is parametrized to handle $2^{P}$ samples in parallel (Parsons 2008). This FFT architecture uses only $25 \%$ more buffering than the theoretical minimum, and still achieves $100 \%$ butterfly utilization efficiency. This feat is achieved by decomposing a $2^{N}$ channel FFT into $2^{P}$ parallel biplex FFTs of length $2^{N-P}$, followed by a $2^{P}$ channel parallel FFT core using time-multiplexed twiddle-factor coefficients.

Finally, we have written modules for performing two real FFTs with each half of a biplex FFT using Hermitian conjugation. Mirroring and conjugating the output spectra to reconstitute the negative frequencies, this module effects a 4-in-1 real biplex FFT that can then be substituted for the equivalent number of biplex cores in a high-bandwidth FFT. Thus, our real FFT module has the same bandwidth flexibility as our standard complex FFT.

Dynamic range inside fixed-point FFTs requires careful consideration. Tones are folded into half as many samples through each FFT stage, causing magnitudes to grow by a factor of 2 for narrow-band signals, and $\sqrt{2}$ for random noise. To avoid overflow and spectrum corruption, our cores contain optional downshifts at each stage. In an interference-heavy environment, one must balance loss of SNR from downshifting signal levels against loss of integration time due to overflows. A good practice is to place time-domain input into the most-significant bits of the FFT and downshift as often as possible to avoid overflow and minimize rounding error in each butterfly 
stage. However, it is also best to avoid using the top 2 bits on input since the first 2 butterfly stages can be implemented using negation instead of complex multiplies, but the asymmetric range of 2's complement arithmetic can allow this negation to overflow.

\subsection{A Cross-Multiplication/Accumulation (X) Engine}

Our FX correlator architecture employs X engines to compute all antenna cross-multiples within a frequency channel, and multiple frequencies are multiplexed into the core as dictated by processor bandwidth; the complex visibility $V_{i j}$ (Eq. 1) is the average of the product of complex voltage samples from antenna $i$ and antenna $j$ with the convention that the voltage $j>i$ is conjugated prior to forming product. In collaboration with Lynn Urry of UC Berkeley's Radio Astronomy Lab we have implemented a parametrized module (Fig. 8) for computing and accumulating all visibilities for a specified number of antennas. An X engine operates by receiving $N_{\text {ant }}$ data blocks in series, each containing $T_{a c c}$ data samples from one frequency channel of one antenna. The first samples of all blocks are cross-multiplied, and the $N_{\text {ant }}\left(N_{\text {ant }}+1\right) / 2$ results are added to the results from the second samples, and so on, until all $T_{a c c}$ samples have been exhausted. Accumulation prevents the data rate out of a cross-multiplier from exceeding the input data rate. An $\mathrm{X}$ engine is divided into stages, each responsible for pairing two different data blocks together: the zeroth stage pairs adjacent blocks, the first stage pairs blocks separated by one, and so on. As the final accumulated results become available, they are loaded onto a shift register and output from the $\mathrm{X}$ engine.

However, as a new window of $N_{\text {ant }} \times T_{a c c}$ samples arrives, some stages, behaving as described above, would compute invalid results using data from two different windows. To avoid this, each stage switches between cross-multiplying separations of $S$ to separations of $N_{\text {ant }}-S$, which happen to be valid precisely when separations of $S$ would be invalid. As a result, there need be only floor $\left(N_{\text {ant }} / 2+1\right)$ stages in an $\mathrm{X}$ engine. Every $T_{a c c}$ samples, each stage outputs a valid result, yielding $N_{\text {ant }} \times$ floor $\left(N_{\text {ant }} / 2+1\right)$ total accumulations; for even values of $N_{\text {ant }}, N_{\text {ant }} / 2$ of the results from the last stage are redundant. All other multiplier/accumulators are 100\% utilized. Each stage also computes all polarization cross-multiples (Eq. 2) using parallel multipliers.

When one $\mathrm{X}$ engine no longer fits on a single FPGA, it may be divided across chips at any stage boundary at the cost of a moderate amount of bidirectional interconnect. The output shift register need not be carried between chips; each FPGA can accumulate and store the results computed locally. In order for the output shift register's floor $\left(N_{\text {ant }} / 2+1\right)$ stages to clear before the next accumulation is ready, an $\mathrm{X}$ engine requires a minimum integration length of: $T_{a c c}>$ floor $\left(N_{\text {ant }} / 2+1\right)$. In current hardware, a practical upper limit on $T_{a c c}$ is set by the $2 \times 4$ Mbit of SRAM storage available on the IBOB. For 2048 channels with 4-bit samples, and double buffering for 2 antennas, 2 polarizations, this limit is $T_{a c c} \leq 128$. Longer integration requires an accumulator capable of buffering an entire vector of visibility data, and typically occurs in off-chip DRAM. The

maximum theoretical accumulation length in correlator is determined by the fringe rate of sources 
moving across the sky, and is a function of observing frequency, maximum antenna separation, and (for correlators with internal fringe rotation) field-of-view across the primary beam.

Cross-multiplication comes to dominate the total correlator processing budget for large numbers of antennas. As a result, care must be taken both to reduce the footprint of a complex multiplier/accumulator and to make full and efficient use of the resources on an FPGA processor. The number of bits used to carry a signal should be minimized while retaining sufficient dynamic range to distinguish signal from noise. We have chosen to focus on 4-bit multipliers in current applications, and the subjects of dynamic equalization and Van Vleck correction generalized to 4 bits are explored in Section 6 for optimizing signal-to-noise ratios (SNR) in our correlators. To make full use of FPGA resources, we construct 4-bit complex multipliers using distributed logic, dedicated multiplier cores, and look-up tables implemented in Block RAMs.

It is possible to perform the bulk of an $N$-bit complex multiply in an $M$-bit multiplier core by sign-extending numbers to $2 N$ bits and combining them into two $M$-bit, unsigned numbers. Multiplying $(a+b i)(c+d i)$, these representations are $\left(2^{M-2 N} a_{s}+b_{s}\right)$ and $\left(2^{M-2 N} c_{s}+d_{s}\right)$, where $n_{s}=2^{2 N}+n$. The bits corresponding to $a c, a d+b c, b d$ may be selected from the product, provided that the sign-extension to $2 N$ bits shifts $a+d$ beyond the bits occupied by $a d$. This yields the constraint:

$$
6 N-1<M
$$

The 18-bit multipliers in current Xilinx FPGAs can efficiently perform 3-bit complex multiplies, but fall short of 4 bits.

\section{System Integration}

\subsection{F Engine Synchronization}

Though we have touted GALS design principles for X engine processing, digitization and spectral processing within $\mathrm{F}$ engines must be synchronized to a time interval much smaller than a spectral window to avoid severe degradation of correlation response (Fig. 9). This attenuation effect, resulting from the changing degree of overlap of correlated signals within a spectral window, can be caused by systematic signal delay between antennas, as well as by source-dependent geometric delay; FX correlators with insufficient channel resolution experience a narrowing of the field of view related to channel bandwidth. This effect has been well explored for FX correlators employing DFTs (see Chapter 8 of Thompson et al. (2001)), but Polyphase Filter Banks show a different response owing to a weighting function that extends well beyond the number of samples

used in a DFT. Given a standard form for PFB sample weighting of sinc $\left(\frac{\pi t}{N \tau_{s}}\right) W\left(\frac{t}{2 T N \tau_{s}}\right)$, where $N$ is the number of output channels, $T$ is the number of PFB taps, $\tau_{s}$ is the delay between timedomain samples, and $W$ is an arbitrary windowing function that tapers to 0 at \pm 1 , the gain versus 
delay $G(\tau)$ of a PFB-based FX correlator is given by:

$$
G(\tau)=\int_{-\infty}^{\infty}\left[\operatorname{sinc}\left(\frac{\pi t}{N \tau_{s}}\right) W\left(\frac{t}{2 T N \tau_{s}}\right)\right] \times\left[\operatorname{sinc}\left(\frac{\pi(t-\tau)}{N \tau_{s}}\right) W\left(\frac{t-\tau}{2 T N \tau_{s}}\right)\right] d t
$$

For the purpose of F Engine synchronization, we rely on a one-pulse-per-second (1PPS) signal with a fast edge-rate provided synchronously to a bank of $F$ processors running off identical system clocks. This signal is sampled by the system clock on each processor, and provided alongside ADC data. A slower, asynchronous "arm" signal is sent from a central node to each F engine at the half second phase to indicate that the next 1PPS signal should be used to generate the reset event that synchronizes spectral windows and packet counters. This ensures that samples from different antennas entering $\mathrm{X}$ engines together were acquired within one or two system clocks of one another. The degree of synchronization is determined by the difference in path lengths of 1PPS and the system clock from their generators to each $\mathrm{F}$ engine. This path length can be determined from celestial source observations using self-calibration, and barring temperature effects, will be constant for a correlator configuration following power-up.

\subsection{Asynchronous, Packetized "Corner Turner"}

The choice of the accumulation length $T_{a c c}$ in $\mathrm{X}$ engines determines the natural size of UDP packets in our packet-switched correlator architecture. For current CASPER hardware where channel-ordering occurs in IBOB SRAM, $T_{a c c}$ is constrained by the available memory to an upper limit of 128 samples for 2048-channel dual-polarization, 4-bit, complex data, yielding a packet payload of 256 bytes. A header containing 2 bytes of antenna index and 6 bytes of frequency/time index is added to each packet to enable packet unscrambling on the receive side. The frequency/time index (hereafter referred to as the master counter, or MCNT) is a counter that is incremented every packet transmission. The lower bits count frequencies within a spectrum, and the rest count time. Combined with the antenna index, MCNT completely determines the time, frequency, source, and destination of each packet; MCNT maps uniquely to a destination IP address.

Packet reception (Fig. 10) is complicated by the realities of packet scrambling, loss, and interference. A circular buffer holding $N_{\text {win }}$ windows worth of $\mathrm{X}$ engine data stores packet data as they arrive. The lower bits of MCNT act as an address for placing payloads into the the correct window, and the antenna index addresses the position within that window. When data arrives $N_{\text {win }} / 2$ windows ahead of a buffered window, that window is flagged for readout, and is processed contiguously on the next window boundary of the free-running $\mathrm{X}$ engine. Using packet arrival to determine when a window is processed allows a data-rate dependent time interval for all packets to arrive, but pushes data through the buffer in the event of packet loss. On readout, the buffer is zeroed to ensure that packet loss results in loss of signal, rather than the introduction of noise. F engines can be intentionally disconnected from transmission without compromising the correlation of those remaining. 
Packet interference occurs when a well-formed packet contains an invalid MCNT as a result of switch latency, unsynchronized F engines, or system misconfiguration. Such packets must be prevented from entering the receive buffer, since they can lead to data corruption; one would prefer that a misconfigured $\mathrm{F}$ engine antenna result in data loss for that antenna, rather than data loss for the entire system. To ensure this behavior, incoming packets face a sliding filter based on currently active MCNTs. Packets are only accepted if their MCNT falls within the range of what can currently be held in the circular buffer. As higher MCNTs are received and accepted, old windows are flagged for read out, freeing up buffer space for still higher MCNTs. This system forces MCNTs to advance by small increments and prevents the large discontinuities indicative of packet interference. In the eventuality that a receive buffer accidentally locks onto an invalid MCNT from the outset, a time-out clause causes the currently active MCNT to be abandoned for a new one if no new data is accepted into the receive buffer.

A final complication comes when implementing a bidirectional 10GbE transmission architecture such as the one outlined in Figure 2. Commercial switches do not support self-addressed packet transmission; they assume that the transmitter (usually a CPU) intercepts these packets and transfers them to the receive buffer. On FPGAs, this requires an extra buffer for holding "loopback", and a multiplexer for inserting these packets into the processing stream. A simple method for this insertion would be to always insert loopback packets, if available, and otherwise to insert packets from the $10 \mathrm{GbE}$ interface. However, there is a maximum interval over which packets with identical MCNTs can be scrambled before the receive system rejects packets for being outside of its buffer. This simple method has the undesirable effect of including switch latency in the time interval over which packets are scrambled, causing unnecessary packet loss. Our solution is to pull loopback packets only after packets with the same MCNT arrive through the switch.

\subsection{Monitor, Control, and Data Acquisition}

The toolflow we have developed for CASPER hardware provides convenient abstractions for interfacing to hardware components such as ADCs, DRAM, and 10 GbE transceivers, and allows specified registers and BRAMs to be automatically connected to CPU-accessible buses. On top of this framework, we run BORPH-an extension of the Linux operating system that provides kernel support for FPGA resources (So \& Brodersen 2006; So 2007). This system allows FPGA configurations to be run in the same fashion as software processes, and creates a virtual file system representing the memories and registers defined on the FPGA. Every design compiled with this toolflow comes equipped with this real-time interface for low- to moderate-bandwidth data I/O. By emulating standard file-I/O interfaces, BORPH allows programmers to use standard languages for writing control software. The majority of the monitor, control, and data acquisition routines in our correlators are written in $\mathrm{C}$ and Python. For 8-16 antenna correlators, the bandwidth through BORPH on a BEE2 board is sufficient to support the output of visibility data with 5-10s integrations. 
For correlators with more antennas or shorter integration times, the bandwidth of the CPU/FPGA interface is incapable of maintaining the full correlator output. This limitation is being overcome by transmitting the final correlator output using a small amount of the extra bandwidth on the $10 \mathrm{GbE}$ ports already attached to each X engine. After accumulation in DRAM, correlator output is multiplexed onto the $10 \mathrm{GbE}$ interface and transmitted to one or more Data Acquisition (DA) systems attached to the central $10 \mathrm{GbE}$ switch. These systems collect and store the final correlator output. With a capable DA system, the added bandwidth through this output pathway can be used to attain millisecond integration times, opening up opportunities for exploring transient events and increasing time resolution for removing interference-dominated data.

The capabilities of correlators made possible by our research are placing new challenges on DA systems (Wright 2005). There is a severe (factor of 100) mismatch between the data rates in the on-line correlator hardware and those supported by the off-line processing. Members of our team are currently pursuing research on how this can be resolved both for correlators and for generic signal processing systems using commercially available compute clusters. For correlators, our group is currently exploring how to implement calibration and imaging in real-time to reduce the burden of expert data reduction on the end user, and to make best use of both telescope and human resources.

\section{Characterization}

\subsection{ADC Crosstalk}

Crosstalk is an undesirable but prevalent characteristic of analog systems wherein a signal is coupled at a low level into other pathways. This can pose a major threat to sensitivity in systems that integrate noise-dominated data to reveal low-level correlation. For CASPER hardware, we have examined crosstalk levels between signal inputs sharing an ADC chip, and between different ADC boards on the same IBOB. Figure 11 illustrates a one-hour integration of uncorrelated noise of various bandwidths input to the "Pocket Correlator" system (see Section 7). Between inputs of the same ADC board, a coupling coefficient of $\sim 0.0016$ indicates crosstalk at approximately -28 $\mathrm{dB}$. This coupling is a factor of 5 higher than the $-35 \mathrm{~dB}$ isolation advertised by the Atmel ADC chip, and is most likely the result of board geometry and shared power supplies. Crosstalk between inputs on different ADCs also peaks at the $-28 \mathrm{~dB}$ level, but shows more frequency-dependent structure.

Crosstalk may be characterized and removed, provided that its timescale for variation is much longer than the calibration interval. Figure 12 demonstrates that for integration intervals ranging from 7.15 seconds to approximately 1 day (the limit of our testing), crosstalk amplitudes and phases vary around stable values in a lab test that, when subtracted, yield noise that integrates down with time. Even though crosstalk is encountered at the $-28 \mathrm{~dB}$ level, its stability allows suppression to at least $-62 \mathrm{~dB}$. This stability has allowed crosstalk to be removed post-correlation, 
and we have until recently deferred adding phase switching. Developments along this line are proceeding by introducing an invertible mixer (controlled via a Walsh counter on an IBOB) early in the analog signal path, and removing this inversion after digitization. Phase switching must be coupled with data blanking near boundaries when the inversion state is uncertain. Blanking will be most easily implemented by intentionally dropping packets of data from $\mathrm{F}$ engine transmission, and by providing a count of results accumulated in each integration for normalization purposes.

\subsection{XAUI Fidelity and Switch Throughput}

CASPER boards are currently configured to transmit XAUI protocol over CX4 ports as a point-to-point communication protocol and as the physical layer of $10 \mathrm{GbE}$ transmission. Because the Virtex-II FPGAs used in current CASPER hardware do not fully support XAUI transmission standards xil (2004, 2005), current devices can have sub-optimal performance for certain cable lengths. We expect the new ROACH board, which employs Virtex-5 FPGAs, to have better performance in this regard. For cable lengths supported in current hardware, we tested XAUI transmission fidelity using matched Linear Feedback Shift Registers (LFSRs) on transmit and receive. Error detection was verified using programmable bit-flips following transmitting LFSRs. Over a period of 16 hours, $573 \mathrm{~Tb}$ of data were transmitted and received on each of 8 XAUI links. During this time, no errors were detected, resulting in an estimated bit-error rate of $2.2 \cdot 10^{-16} \mathrm{~Hz}$. We also tested the capability of two Fujitsu switches (the XG700 and the XG2000) for performing the full cross-connect packet switching required in our FX correlator architecture. By tuning the sample rate inside $\mathrm{F}$ engines of an 8-antenna (4-IBOB) packetized correlator, we controlled the transmission rate per switch port over a range of 5.96 to $8.94 \mathrm{~Gb} / \mathrm{s}$. In 10-minute tests, packet loss was zero for both switches in all but the $8.94 \mathrm{~Gb} / \mathrm{s}$ case. Packet loss in this final case was traced to intermittent XAUI failure as a result of imperfect compliance with the XAUI standard, as described previously. Overheating of FPGA chips in the field has also been reported as a source of intermittent operation.

\subsection{Equalization and 4-Bit Requantization}

Correlator processing resources can be reduced by limiting the bit width of frequency-domain antenna data before cross-multiplication. However, digital quantization requires careful setting of signal levels for optimum SNR and subsequent calibration to a linear power scale (Thompson et al. 2001; Jenet \& Anderson 1998). Correlators using 4 bits represent an improvement over their 1 and 2 bit predecessors, but there are still quantization issues to consider. The total power of a 4-bit quantizer has a non-linear response with respect to input level as shown in Figure 13 . In currently deployed correlators, we perform equalization (per channel scaling) to control the RMS channel values before requantizing from 18 bits to 4 bits. This operation saturates RFI and flattens the passband to reduce dynamic range and to hold the passband in the linear regime of the 4-bit 
quantization power curve. Equalization is implemented as a scalar multiplication on the output of each PFB using 18-bit coefficients from a dynamically updateable memory. These coefficients allow for automatic gain control to maintain quantization fidelity through changing system temperatures.

\section{Deployments and Results}

\subsection{A Pocket Correlator}

The "Pocket Correlator" (Fig. 14) is a single IBOB system that includes F and X engines on a single board for correlating and accumulating 4 input signals. Each input is sampled at 4 times the FPGA clock rate (which runs up to $250 \mathrm{MHz}$ ), and a down-converter extracts half of the digitized band. This subband is decomposed into 2048 channels by an 8-tap PFB, equalized, and requantized to 4 bits. With all input signals on one chip, X processing can be implemented directly as multipliers and vector accumulators, rather than as $\mathrm{X}$ engines. Limited buffer space on the IBOB permits only 1024 channels (selectable from within the 2048) to be accumulated. Output occurs either via serial connection (with a minimum integration time of 5 seconds) or via 100-Mbit UDP transmission (with a minimum integration time in the millisecond range). This system can act as a 2-antenna, full Stokes correlator, or as a 4-antenna single polarization correlator.

The Pocket Correlator is valuable as a simple, stand-alone instrument, and for board verification in larger packetized systems. It is being applied as a stand-alone instrument in PAPER, the ATA, and the UNC PARI observatory. A 4-antenna, single polarization deployment of the PAPER experiment in Western Australia in 2007 used the Pocket Correlator to collect the data used to produce a $150 \mathrm{MHz}$ all-sky map illustrated in Figure 15. In addition to demonstrating the feasibility of post-correlation crosstalk removal, this map (specifically, the imperfectly removed sidelobes of sources) illustrates a problem that will require real-time imaging to solve for large numbers of antennas.

\subsection{An 8-Antenna, 2-Stokes, Synchronous Correlator}

This first generation multi-board correlator demonstrated the functionality of signal processing algorithms and CASPER hardware, but preempted the current packetized architecture-it operated synchronously. This version of the correlator was most heavily limited by $\mathrm{X}$ engine resources, all

of which were implemented on a single FPGA to simplify interconnection. The total number of complex multipliers in the $\mathrm{X}$ engines of an $N_{\text {ant }}$ antenna array is: $N_{c m a c}=\operatorname{floor}\left(N_{\text {ant }} / 2+1\right) \times$ $N_{a n t} \times N_{\text {pol }}$; the limited number of multipliers on a BEE2 FPGA only allowed for supporting half the polarization cross-multiples. This system was an important demonstration of the basic capabilities of our hardware and software, and provided a starting point for evolving a more sophisticated system. Deployments of this system at the NRAO site in Green Bank as part of the PAPER 
experiment, and briefly at the Hat Creek Radio Observatory for the Allen Telescope Array, are being supersede by the packetized correlator presented in the next section.

\subsection{A 16-Antenna, Full-Stokes, Packetized Correlator}

This packetized FX correlator is a realization of the architecture outlined in Figure 2, with F processing for 2 antennas implemented on each IBOB, and matching $\mathrm{X}$ processors implemented on each corner FPGA of two BEE2s. Each F processor is identical to a Pocket Correlator (Fig. 14), but branches data from the equalization module to a matrix transposer in IBOB SRAM to form frequency-based packets. Packet data for each antenna are multiplexed through a point-to-point XAUI connection to a BEE2-based X processor, and then relayed in 10GbE format to the switch. The number of channels in this system is limited to 2048 by memory in IBOB SRAM for transposing the 128 spectra needed to meet bandwidth restrictions between X engines and DRAM-based vector accumulators.

The $\mathrm{X}$ processor in this packetized correlator implements the transmit and receive architecture illustrated in Figure 16 for two X engines sharing the same 10GbE link. Each X engine's data processing rate is determined by packets arriving in its own receive buffer, and results are accumulated in separate DRAM DIMMs. The accumulated output of each X processor is read out of DRAM at a low bandwidth and transmitted via $10 \mathrm{GbE}$ packets to a CPU-based server where all visibility data is collected and written to disk in MIRIAD format (Sault et al. 1995) using interfaces from the Astronomical Interferometry in PYthon (AIPY) packag $\AA^{5}$.

The clocks for the BEE2 FPGAs are asynchronous 200-MHz oscillators, and IBOBs run synchronously at any rate lower than this. Packet transmission is statically addressed so that all each $\mathrm{X}$ engine processes every 16th channel. We use 8 ports of a Fujitsu XG700 switch to route data. This system is is scalable to 32 antennas before two $\mathrm{X}$ engines no longer fit on a single FPGA. For larger systems, the number of BEE2s will scale as the square of the number of antennas, and the number of IBOBs will scale linearly. A 32-antenna, 200-MHz correlator on 16 IBOBs and 4 BEE2s is now working in the lab, and a 16-antenna version using 8 IBOBs and 2 BEE2s has been deployed to the NRAO site in Green Bank with the PAPER experiment.

\section{Conclusion}

By decreasing the time and engineering costs of building and upgrading correlators, we aim to reduce the total cost of correlators for a wide range of scales. Small- and medium-scale correlators with total cost dominated by development clearly stand to benefit from our research. It is less clear

\footnotetext{
${ }^{5}$ http://pypi.python.org/pypi/aipy
} 
if the cost of large-scale correlators can be reduced by the general-purpose hardware used in our architecture. Though minimization of replication cost favors the development of specialized parts, there are two factors that can make a generic, modular solution cost less.

The first factor to consider is time to deployment. Even if the monetary cost of development is negligible in the budget of a large correlator, the cost of development time can be significant. If a custom solution takes several years to go from design to implementation, the hardware that is deployed will be out of date. Moore's Law suggests that when a custom solution taking 3 years to develop is deployed, there will exist processors 4 times more powerful, or 4 times less expensive for the equivalent system. The cost of a generic, modular system has to be tempered by the expected savings of committing to hardware closer to the ultimate deployment date.

The second factor is the cost of upgrade. Many facilities (including the ATA) are beginning to appreciate the advantages of designing arrays with wider bandwidths and larger numbers of antennas than can be handled by current technology. Correlators may then be implemented inexpensively on scales suited to current processors, and upgraded as more powerful processors become available. Modular solutions facilitate this methodology.

This and other CASPER research are supported by the National Science Foundation Grant No. 0619596 for Low Cost, Rapid Development Instrumentation for Radio Telescopes. We would like to acknowledge the students, faculty and sponsors of the Berkeley Wireless Research Center, and the National Science Foundation Infrastructure Grant No. 0403427. Correlator development for the PAPER project is supported by NSF grant AST-0505354, and for the ATA project by NSF grant AST-0321309 as well as the Paul G. Allen Foundation. Chips and software were generously provided by Xilinx, Inc. JM and PM gratefully acknowledge financial support from the MeerKAT project and South Africa's National Research Foundation.

Glossary of Technical Terms

- ADC - Analog to Digital Converter

- ASIC - Application-Specific Integrated Circuit processor

- BEE2 - Berkeley Emulation Engine, rev. 2

- BORPH - Berkeley Operating system for Re-Programmable Hardware

- BRAM - Block RAM: Random Access Memory inside an FPGA

- CX4 - 10GbE-compatible industry standard connector

- CPU - Central Processing Unit

- DDR2 - Double-Data-Rate 2 type of off-FPGA Synchronous DRAM 
- DIMM - Dual Inline Memory Module

- DFT - Discrete Fourier Transform

- DRAM - Dynamic Random Access Memory

- FFT - Fast Fourier Transform algorithm

- FIR - Finite Impulse Response digital filter

- FPGA - Field Programmable Gate Array processor

- FX - Correlator architecture implemented as frequency channelization, then cross-multiplication

- GALS - Globally Asynchronous, Locally Synchronous system architecture

- GB - GigaByte

- IBOB - Internet Break-Out Board

- LFSR - Linear Feedback Shift Register

- LO - Local Oscillator

- MCNT - Master Counter

- PFB - Polyphase Filter Bank

- PowerPC - a specific CPU architecture

- QDR - Quad-Data-Rate type of off-FPGA SRAM

- ROACH - Reconfigurable, Open Architecture for Computing Hardware

- SNR - Signal-to-Noise Ratio

- SRAM - Static Random Access Memory

- UDP - User Datagram Protocol Ethernet packetization

- XAUI - X (ten) Attachment Unit Interface point-to-point transmission protocol

- XF - Correlator architecture implemented as cross-multiplication, then frequency channelization

- 1PPS - 1 Pulse Per Second clock signal

- 10GbE - 10 Gigabit per second Ethernet communication standard 


\section{REFERENCES}

2004, RocketIO Tranceiver User Guide (UG024 V2.5), Xilinx user guide, http://www.xilinx.com

2005, Virtex-II Pro and Virtex-II Pro X Platform FPGAs: Functional Description (DS083-2 V4.5), Xilinx data sheet, http://www.xilinx.com

Blackman, R. \& Tukey, J. 1958, The measurement of power spectra (Dover Publications Inc.)

Bradley, R., Backer, D., Parsons, A., Parashare, C., \& Gugliucci, N. E. 2005, in Bulletin of the American Astronomical Society, 1216-+

Chang, C., Wawrzynek, J., \& Brodersen, R. W. 2005, IEEE Design and Test of Computers, 22, 114

Chapiro, D. M. 1984, PhD thesis, Stanford Univ., CA.

Crochiere, R. \& Rabiner, L. R. 1983, Multirate Digital Signal Processing (Englewood Cliffs, N.J., Prentice-Hall, Inc., 1983. 336 p.)

D'Addario, L. 2001, ATA Memo

Demorest, P., Ramachandran, R., Backer, D., Ferdman, R., Stairs, I., \& Nice, D. 2004, in Bulletin of the American Astronomical Society, 1598-+

Dick, C. 2000, Xilinx Application Note

Heiles, C., Goldston, J., Mock, J., Parsons, A., Stanimirovic, S., \& Werthimer, D. 2004, in Bulletin of the American Astronomical Society, 1476-+

Jenet, F. A. \& Anderson, S. B. 1998, PASP, 110, 1467

Parsons, A. 2008, IEEE Signal Processing Letters, submitted

Parsons, A., Backer, D., Chang, C., Chapman, D., Chen, H., Crescini, P., de Jesus, C., Dick, C., Droz, P., MacMahon, D., Meder, K., Mock, J., Nagpal, V., Nikolic, B., Parsa, A., Richards, B., Siemion, A., Wawrzynek, J., Werthimer, D., \& Wright, M. 2006, in Asilomar Conference on Signals and Systems, Pacific Grove, CA, 2031-2035

Plana, L. A., Furber, S. B., Temple, S., Khan, M., Shi, Y., Wu, J., \& Yang, S. 2007, IEEE Des. Test, 24, 454

Rabiner, L. R. \& Gold, B. 1975, Theory and application of digital signal processing (Englewood Cliffs, N.J., Prentice-Hall, Inc., 1975. 777 p.)

Rybicki, G. B. \& Lightman, A. P. 1979, Radiative processes in astrophysics (New York, WileyInterscience, 1979. 393 p.) 
Sault, R. J., Teuben, P. J., \& Wright, M. C. H. 1995, in Astronomical Society of the Pacific Conference Series, Vol. 77, Astronomical Data Analysis Software and Systems IV, ed. R. A. Shaw, H. E. Payne, \& J. J. E. Hayes, 433-+

So, K. H. 2007, PhD thesis, Berkeley Wireless Research Center, UC Berkeley, CA.

So, K. H. \& Brodersen, R. W. 2006, in 16th International Conference on Field Programmable Logic and Applications, 349-354

Thompson, A. R., Moran, J. M., \& Swenson, Jr., G. W. 2001, Interferometry and Synthesis in Radio Astronomy, 2nd Edition (New York, Wiley-Interscience, 2001. 692 p.)

Vaidyanathan, P. P. 1990, in IEEE, Vol. 78, 56-93

Weinreb, S. 1961, Proc. IEEE, 49, 1099

Wright, M. 2005, SKA Memo

Yen, J. L. 1974, A\&AS, 15, 483 


\begin{tabular}{lrrrr}
\multicolumn{4}{c}{ Price and Power Consumption of CASPER Hardware } \\
\hline \hline Board & Board & Cost with & Gops & Power \\
& Cost & FPGAs & per Sec & $(\mathrm{W})$ \\
\hline IBOB & $\$ 400$ & $\$ 2700$ & 70 & 30 \\
BEE2 & $\$ 5000$ & $\$ 23500$ & 500 & 150 \\
ROACH* & $\$ 1000$ & $\$ 3200$ & 400 & 50 \\
ADC $(1 \mathrm{Gs} / \mathrm{s} \times 2)$ & $\$ 200$ & $\$ 200$ & $\mathrm{~N} / \mathrm{A}$ & 2 \\
ADC $(3 \mathrm{Gs} / \mathrm{s})^{*}$ & $\$ 1000$ & $\$ 1000$ & $\mathrm{~N} / \mathrm{A}$ & 5 \\
\hline \hline
\end{tabular}

*Estimated from prototype versions.

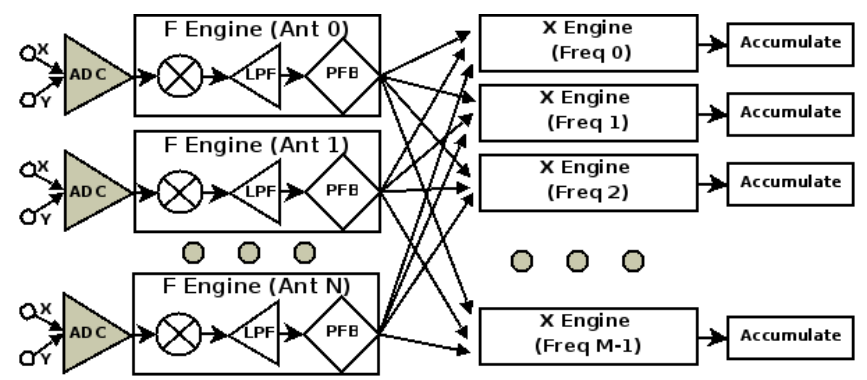

Fig. 1.- In a simplistic FX correlator, the signals from $\mathrm{N}$ antennas are first decomposed into $\mathrm{M}$ frequency channels (F operation) and then cross-multiplied (X operation). Different channels are never cross-multiplied, making them natural units for $\mathrm{X}$ engine processing. Thus, each $\mathrm{X}$ engine handles all baselines for one frequency channel.

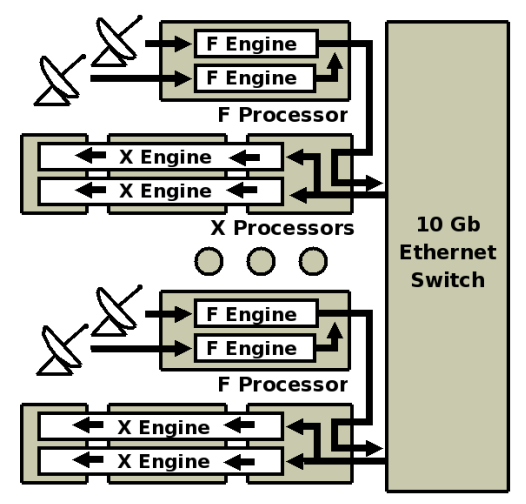

Fig. 2.- Data bandwidth per antenna is equal to the processing bandwidth of an $\mathrm{X}$ processor in this example application. Transmitted data is routed through an $\mathrm{X}$ processor to take advantage of bidirectionality of $10 \mathrm{GbE}$ ports, thereby halving the number of ports on the switch. 


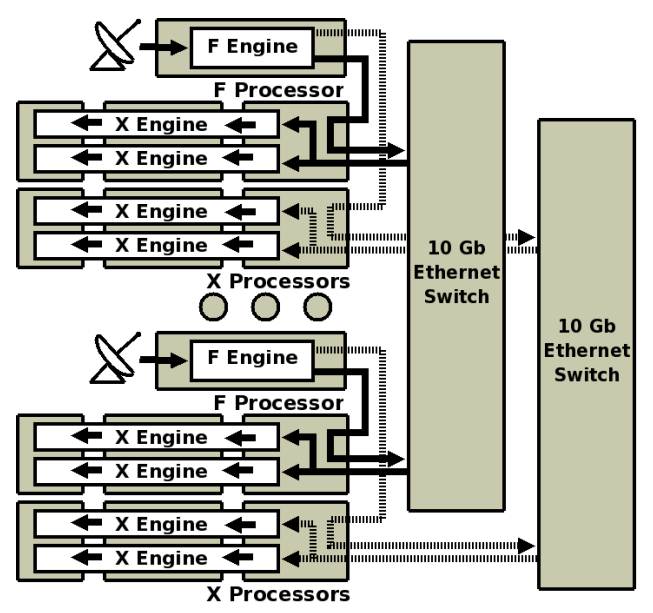

Fig. 3.- Data bandwidth per antenna can exceed what can be carried over 10GbE. Here, the frequency band has been spread across ports by channel, so that each half of transmission occurs on an isolated subnet. This is possible because different channels are never cross-multiplied in an FX correlator.

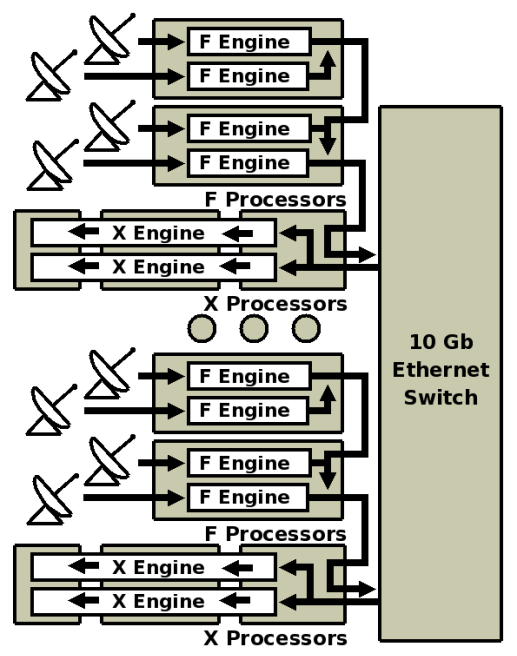

Fig. 4.- When the processing bandwidth of an X engine exceeds the antenna bandwidth by at least a factor of 2 , half as many $\mathrm{X}$ processors are needed for a given number of antennas. $\mathrm{X}$ processors operate independently of data bandwidth; the same design handles this and the previous two cases (Figs. 2 and 3). Only the number of X processors and the data transmission pattern have changed. 


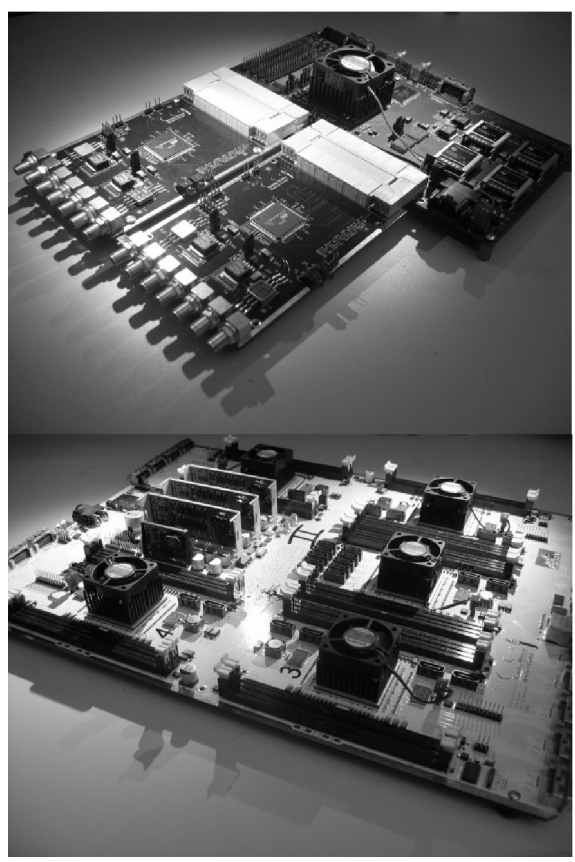

Fig. 5.- Our correlator architecture relies on modular FPGA-based processing hardware developed by our group to combine flexibility, upgradeability, and performance. Illustrated above are: (top) IBOB and ADC FPGA/digitizer modules (bottom) The Berkeley Emulation Engine (BEE2) FPGA board

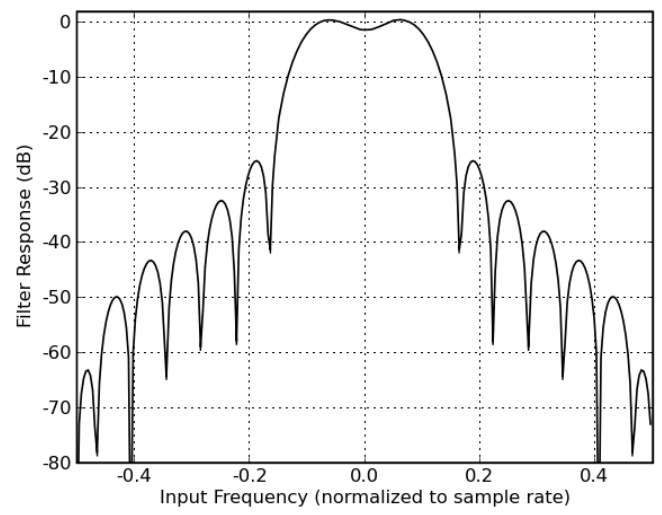

Fig. 6.- This example response an the FIR filter in a digital down-converter, illustrates the 16 tap low-pass design used in the correlator deployments presented later. 


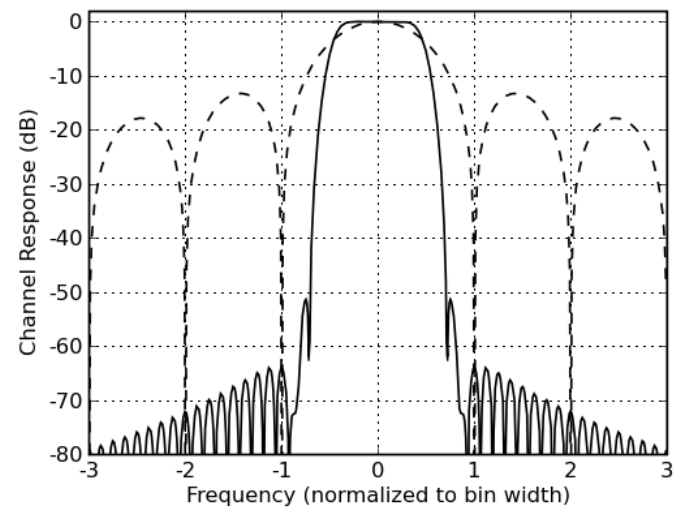

Fig. 7.- The response of a frequency channel in an 8-tap Polyphase Filter Bank (solid) using a Hamming window is compared to an equivalently sized Discrete Fourier Transform (dashed). This particular PFB, implemented for 2048 channels, is used in the correlator deployments presented in Section 7 .

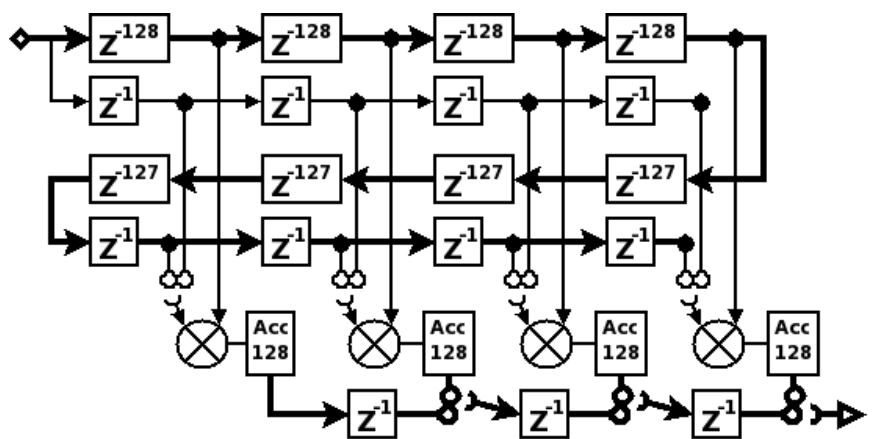

Fig. 8.- This $\mathrm{X}$ engine schematic illustrates the pipelined flow of data that allows it to be split across multiple FPGAs and boards. With continuous data input, all multipliers (with the possible exception of the final stage for even values of $N_{\text {ant }}$ ) are used with $100 \%$ efficiency. 


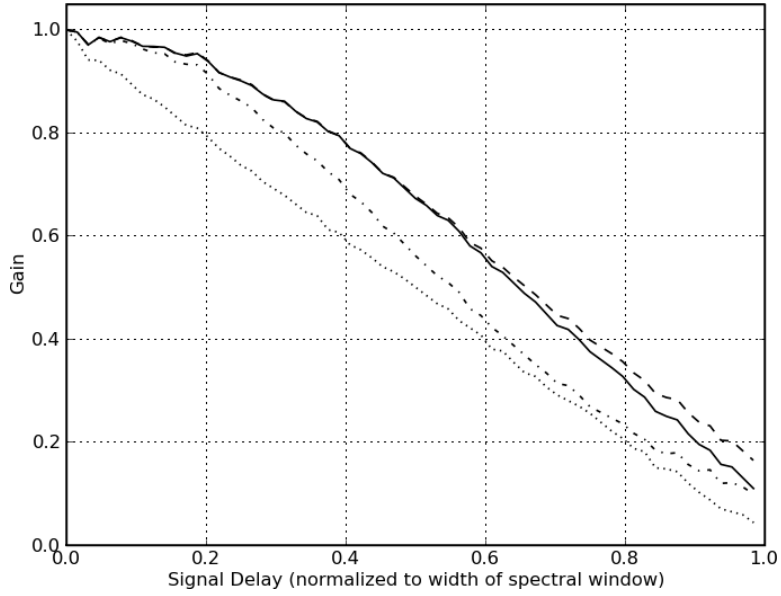

Fig. 9. - Cross-correlation of noise decreases as a function of signal delay between antenna inputs. PFBs operate on a wider window of data compared to DFTs, and use non-flat sample weightings, yielding a different correlation response versus signal delay compared to the standard result presented in Thompson et al. (2001) Thompson et al. (2001). Graphed are the responses of PFBs with 8 taps (solid), 4 taps (dashed), 2 taps (dot dashed), and the response of a DFT (dotted).

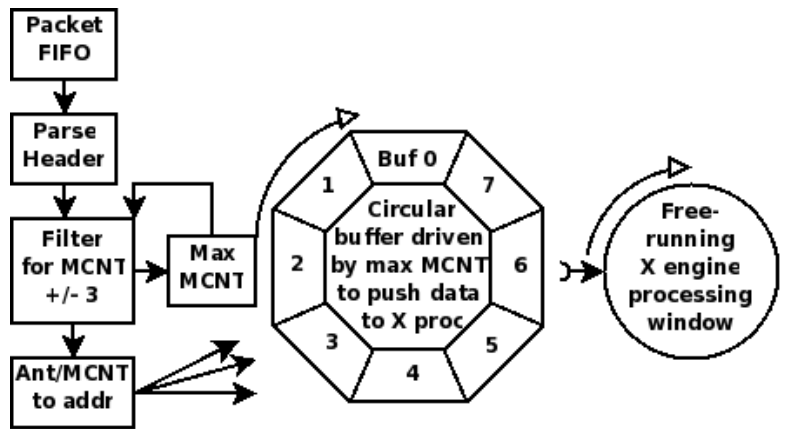

Fig. 10. - Before transmission, each $\mathrm{F}$ engine packet is tagged with an antenna number and master counter (MCNT) encoding time and frequency. Received packets are filtered to the narrow range of MCNTs, and maximum MCNT slides smoothly up as packets are received. A free-running X engine processes available windows when it is ready. This architecture allows data to be processed at a lower data rate than the FPGA clock rate without requiring every element in the pipeline to have a enable signal. 


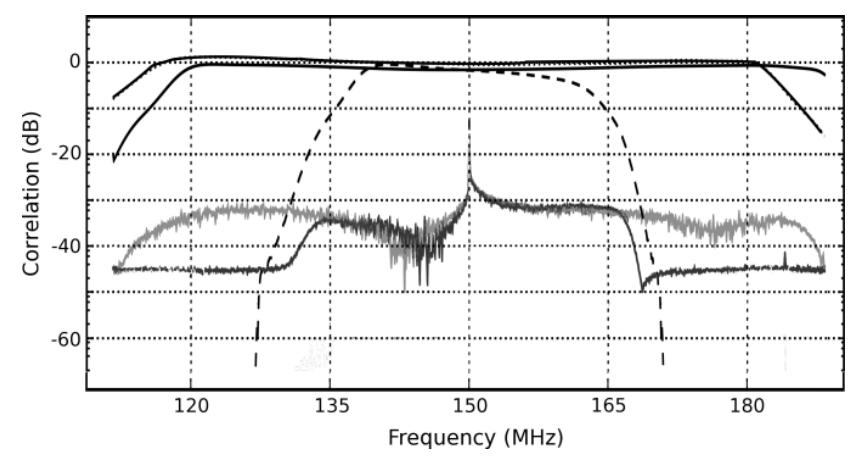

Fig. 11. - Uncorrelated noise sources with similar bandpass shapes were input to two channels of one ADC board (solid black) and a third noise source with a narrower passband was input to to a second ADC board (dashed black) in the "Pocket Correlator" system. Crosstalk levels between signal inputs on the same ADC board (light gray) and between ADC boards sharing an IBOB (dark gray) peak at $-28 \mathrm{~dB}$.

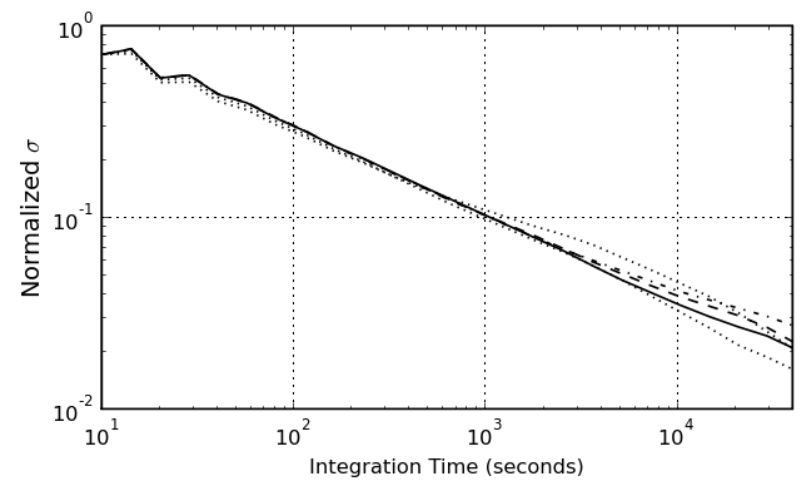

Fig. 12.- Measurements of the standard deviation versus integration time of the correlation between independent noise sources into the same ADC board show that crosstalk exhibits stability over a period of 1 day for all frequency channels Although phase switching may still be desireable, this stability allows crosstalk to be calibrated and removed after correlation. 


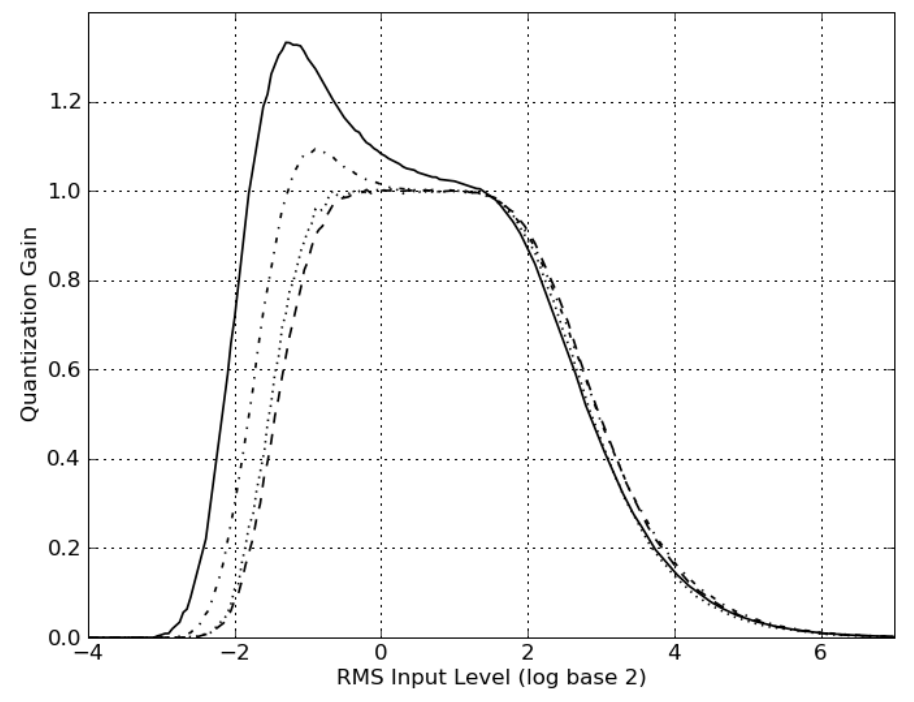

Fig. 13.- Illustrated above is the relative gain through a 4-bit, 15-level quantizer as a function of input signal level (log base 2). Plotted are gain curves for the cross-correlation of two gaussian noise sources with correlation levels of $100 \%$ (solid), $80 \%$ (dot-dashed), $40 \%$ (dotted), and $20 \%$ (dashed).

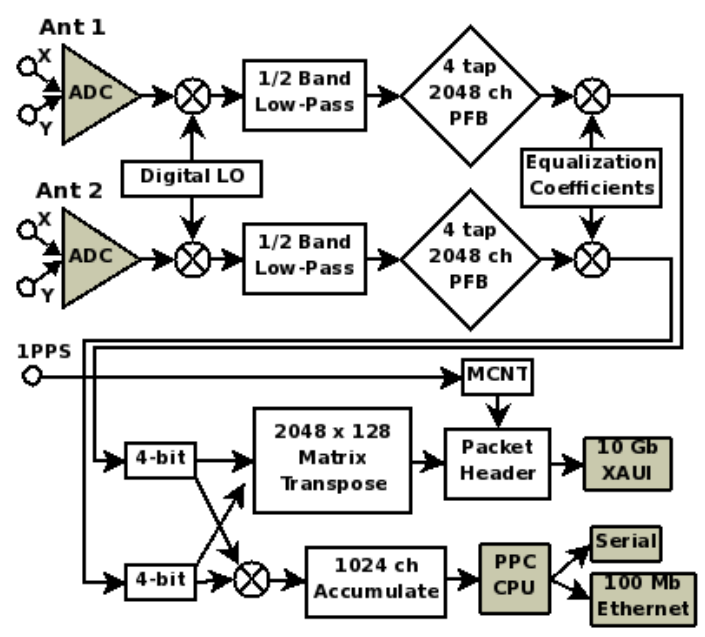

Fig. 14.- This IBOB design serves a dual purpose as a stand-alone "Pocket Correlator" and an F processor in a 16 antenna packetized correlator deployment. Note the parallel output pathways for each function. 


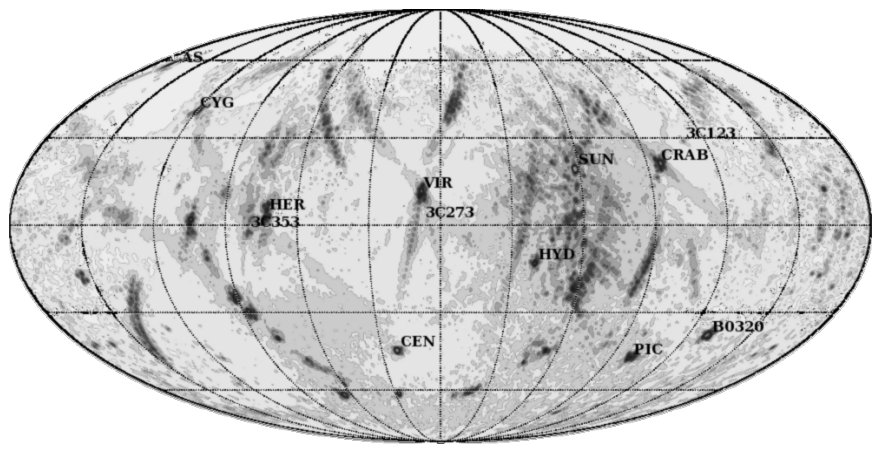

Fig. 15. - This all-sky image, made using a 75-MHz band centered at $150 \mathrm{MHz}$ with the "Pocket Correlator" as part of the PAPER experiment in Western Australia, achieves an impressive 10,000:1 signal-to-noise ratio using 1 day of data.

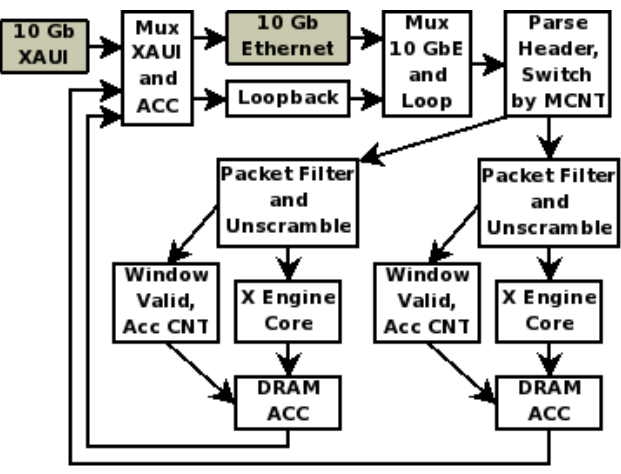

Fig. 16. - A BEE2-based $\mathrm{X}$ processor in a packetized correlator transmits data from an $\mathrm{F}$ engine over $10 \mathrm{GbE}$ and stores self-addressed packets in a "loopback" buffer. These streams are merged on the receive side, and packets are distributed to two X engines. Accumulation occurs in DRAM buffers, and the results are packetized and output over the same 10GbE link. A data aquisition system connects to the same switch as the $\mathrm{X}$ engines. 